\title{
Energy-based modelling and simulation of the interconnection of a back-to-back converter and a doubly-fed induction machine*
}

\author{
Carles Batlle \\ MAIV, EPSEVG and IOC, \\ Univ. Poli. de Catalunya \\ 08800 Vilanova i la G., Spain \\ carles. bat1le@upc. edu
}

\author{
Arnau Dòria-Cerezo \\ DEE, EPSEVG and IOC, \\ Univ. Poli. de Catalunya \\ 08800 Vilanova i la G., Spain \\ arnau.doria@upc. edu
}

\begin{abstract}
This paper describes the port interconnection of two subsystems: a power electronics subsystem (a back-to-back AC/AC converter (B2B), coupled to a phase of the power grid), and an electromechanical subsystem (a doubly-fed induction machine (DFIM). The B2B is a variable structure system (VSS), due to the presence of control-actuated switches; however, from a modelling and simulation, as well as a control-design, point of view, it is sensible to consider modulated transformers (MTF in the bond graph language) instead of the pairs of complementary switches. The port-Hamiltonian models of both subsystems are presented and, using a power-preserving interconnection, the Hamiltonian description of the whole system is obtained; detailed bond graphs of all subsystems and the complete system are also provided. Using passivity-based controllers computed in the Hamiltonian formalism for both subsystems, the whole model is simulated; simulations are run to test the correctness and efficiency of the Hamiltonian network modelling approach used in this work.
\end{abstract}

\section{INTRODUCTION}

The central paradigm of network modelling of complex systems is to have individual open subsystems with well defined port interfaces, hiding an internal model of variable complexity, and a set of rules describing how the subsystems interact through the port variables.

One implementation of this general idea is what is known as port Hamiltonian systems or port-controlled Hamiltonian systems (PCHS) [1][2] (see also [3] and references therein). In this approach, energy plays a fundamental role, port variables are conjugated variables such that their product has dimension of power, and the interconnection of subsystems is implemented by means of what is called a Dirac structure, which enforces the preservation of power, and can be seen as a generalization of Tellegen's theorem of circuit theory [4]. PCHS theory allows the coupling of systems from different domains using energy as the linking concept, and provides the mathematical foundation for bondgraph modelling [5][6]. Although originally developed for lumped parameter systems, PCHS theory has been extended to distributed parameter systems as well [7].

Besides describing systems in a modular, scalable and non domain-specific way, PCHS theory allows a natural implementation of passivity-based control methods [8][9],

\footnotetext{
*This work has been done in the context of the European sponsored project Geoplex with reference code IST-2001-34166 (further information is available at http://www.geoplex.cc), and partially supported by the spanish project DPI2004-06871-CO2-02.
}

using energy as the storage function. The clear separation between (a) constitutive relations, given by the energy, or Hamiltonian, function, (b) the structure matrix, describing how energy flows inside the system, and (c) the power ports, some of which may be terminated by dissipative elements, allows the design of controllers with a clear physical interpretation in what is known as Interconnection and Damping Assignment Passivity-Based Control (IDA-PBC) [10].

Bond graphs [11] can be seen as a graphical representation of the PCHS. A bond graph is a graphical, networkbased description of a physical system and describes the energy interconnection between its generating, storing and dissipating elements. The bond graph description permits the integration of submodels easily and, by means of a simple computer algorithm, the simulation-ready equations of a complex model can be obtained.

In this paper, bond graph and PCHS models of a complex system, see Figure 1, obtained from the interconnection of a doubly-fed induction machine (DFIM) and a back-to-back (B2B) power converter, are presented. Simulations in closed loop, with a control designed by means of Hamiltonian passive techniques, are also performed.

Doubly-fed induction machines have been proposed in the literature, among other applications, for high performance storage systems [12], wind-turbine generators [13] or hybrid engines [14]. The attractiveness of the DFIM stems primarily from its ability to handle large speed variations around the synchronous speed (see [15] for an extended literature survey and discussion).

The back-to-back converter, connected to an auxiliary single-phase grid, provides the desired PWM rotor voltages to the DFIM. The B2B has the nice feature that power can flow in any direction. In particular, in our application the rotor energy of the DFIM can flow back to the converter for some operating conditions [12].

The paper is organized as follows. Section II presents the port Hamiltonian and bond graph models of the several subsystems and their interconnection, and the Dirac structures involved in the interconnection and some associated transformations are identified. Using these, both a port Hamiltonian and a bond graph models of the full system are constructed. Section III displays simulations of the closed loop system for several operating conditions, and Section IV states our conclusions. 


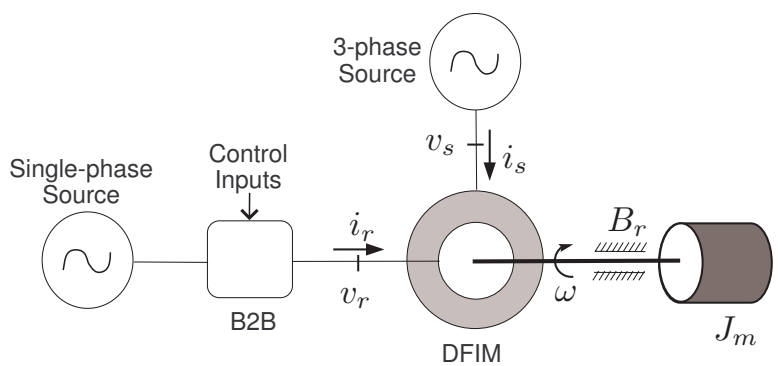

Fig. 1. The system: A DFIM controlled by a B2B converter.

\section{BOND GRAPH AND PORT-CONTROLLED HAMILTONIAN MODELS}

Bond graph theory is a powerful technique to model physical systems [11]. This technique is graphically oriented and represents the power flow between the different elements of a system.

Mathematical equations suitable for simulation can be easily deduced, either manually or using specialized software, from the bond graph representation. This represents a big advantage for complex and/or large systems made of smaller subsystems, since the bond graph description is inherently modular and scalable.

Port-controlled Hamiltonian systems (PCHS) can be seen as a mathematical description of bond graphs [6]. An explicit PCHS has the form [3]

$$
\left\{\begin{array}{l}
\dot{x}=(\mathcal{J}(x)-\mathcal{R}(x))(\nabla H(x))^{T}+g(x) u \\
y=g^{T}(x)(\nabla H(x))^{T}
\end{array}\right.
$$

where $x \in \mathbb{R}^{n}$ are the energy variables, $H(x): \mathbb{R}^{n} \rightarrow \mathbb{R}$ is the energy (or Hamiltonian) function, $u, y \in \mathbb{R}^{m}$ are the port variables, $\mathcal{J}(x)=-\mathcal{J}^{T}(x) \in \mathbb{R}^{n \times n}$ is the intra-connection structure matrix, describing how the energy flows inside the system, $\mathcal{R}=\mathcal{R}^{T} \geq 0 \in \mathbb{R}^{n \times n}$ is the dissipation matrix, and $g(x) \in \mathbb{R}^{n \times m}$ is the interconnection matrix, describing the port connection of the system to the outside world. Port variables are conjugated, so that $[u][y]$ has units of power. Non-negativeness of $\mathcal{R}$ ensures that the map $u \rightarrow y$ is passive.

\section{A. The Doubly-fed Induction Machine}

A Port-controlled Hamiltonian model of a DFIM is given in [12]. This model is described in $d q$-coordinates [16], so that three-phase variables $(a b c)$ are reduced to two-phase variables $(d q)$. The variables are (the $D$ subindex refers to the DFIM subsystem) $x_{D}^{T}=\left(\lambda_{s}^{T}, \lambda_{r}^{T}, J_{m} \omega_{r}\right) \in \mathbb{R}^{5}$, or $x_{D}^{T}=\left(\Lambda^{T}, x_{m}\right)$, where $\Lambda^{T}=\left(\lambda_{s}^{T}, \lambda_{r}^{T}\right) \in \mathbb{R}^{4}, \lambda_{s}, \lambda_{r} \in \mathbb{R}^{2}$ are the inductor fluxes in $d q$-coordinates (stator and rotor respectively), $x_{m}=J_{m} \omega_{r}$ is the mechanical Hamiltonian variable, $\omega_{r}$ the angular speed of the rotor, and $J_{m}$ is the total moment of inertia of the rotating parts. The structure $\mathcal{J}_{D} \in \mathbb{R}^{5 \times 5}$ and damping $\mathcal{R}_{D} \in \mathbb{R}^{5 \times 5}$ matrices are

$$
\mathcal{J}_{D}=\left(\begin{array}{ccc}
-\omega_{s} L_{s} J_{2} & -\omega_{s} L_{s r} J_{2} & O_{2 \times 1} \\
-\omega_{s} L_{s r} J_{2} & -\left(\omega_{s}-\omega_{r}\right) L_{r} J_{2} & L_{s r} J_{2} i_{s} \\
O_{1 \times 2} & L_{s r} i_{s}^{T} J_{2} & 0
\end{array}\right)
$$

$$
\mathcal{R}_{D}=\left(\begin{array}{ccc}
R_{s} I_{2} & O_{2 \times 2} & O_{2 \times 1} \\
O_{2 \times 2} & R_{r} I_{2} & O_{2 \times 1} \\
O_{1 \times 2} & O_{1 \times 2} & B_{r}
\end{array}\right)
$$

where $L$ are inductances, $R$ are resistances, lower indices $s$ and $r$ refer to stator and rotor respectively, $B_{r}$ is the mechanical damping, $i_{s}$ and $i_{r} \in \mathbb{R}^{2}$ are the stator and rotor currents and

$$
J_{2}=\left(\begin{array}{cc}
0 & -1 \\
1 & 0
\end{array}\right) \in \mathbb{R}^{2 \times 2} \quad I_{2}=\left(\begin{array}{ll}
1 & 0 \\
0 & 1
\end{array}\right) \in \mathbb{R}^{2 \times 2} .
$$

Currents $i^{T}=\left(i_{s}^{T}, i_{r}^{T}\right) \in \mathbb{R}^{4}$ and fluxes $\Lambda$ are related by $\Lambda=\mathcal{L} i$, where the inductance matrix $\mathcal{L}$ is

$$
\mathcal{L}=\left(\begin{array}{cc}
L_{s} I_{2} & L_{s r} I_{2} \\
L_{s r} I_{2} & L_{r} I_{2}
\end{array}\right) \in \mathbb{R}^{4 \times 4} .
$$

The interconnection matrix is

$$
g_{D}=\left(\begin{array}{cc}
I_{2} & O_{2 \times 2} \\
O_{2 \times 2} & I_{2} \\
O_{1 \times 2} & O_{1 \times 2}
\end{array}\right) \in \mathbb{R}^{5 \times 4}
$$

with the port variables $u^{T}=\left(v_{s}^{T}, v_{r}^{T}\right) \in \mathbb{R}^{4}$, where $v_{s}, v_{r} \in$ $\mathbb{R}^{2}$ are the stator and rotor voltages. Finally, the Hamiltonian function is

$$
H_{D}=\frac{1}{2} \Lambda^{T} \mathcal{L}^{-1} \Lambda+\frac{1}{2 J_{m}} x_{m}^{2} .
$$

The bond graph of the DFIM is depicted in Fig. 2.

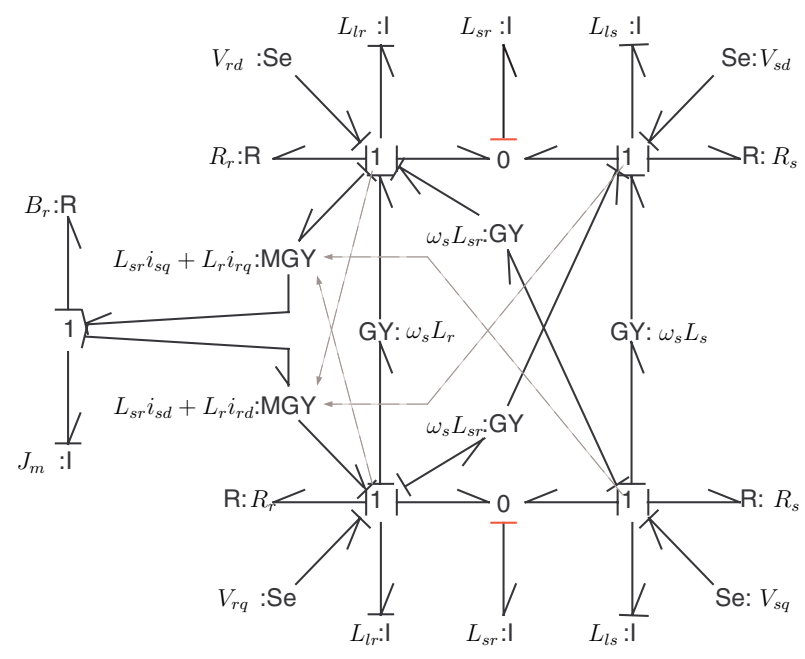

Fig. 2. Bond Graph of the DFIM.

\section{B. The back-to-back converter}

Fig. 3 shows the back-to-back converter selected for this system. It is made of a full bridge AC/DC single-phase boost-like rectifier and a 3-phase DC/AC inverter. The whole converter has an AC single-phase voltage input and its output are 3-phase PWM voltages which feed the rotor windings of the electrical machine. This system can be split into two parts: a dynamical subsystem (the full bridge rectifier, containing the storage elements), and an static subsystem (the inverter, which, from the energy point of view, acts like a transformer). 


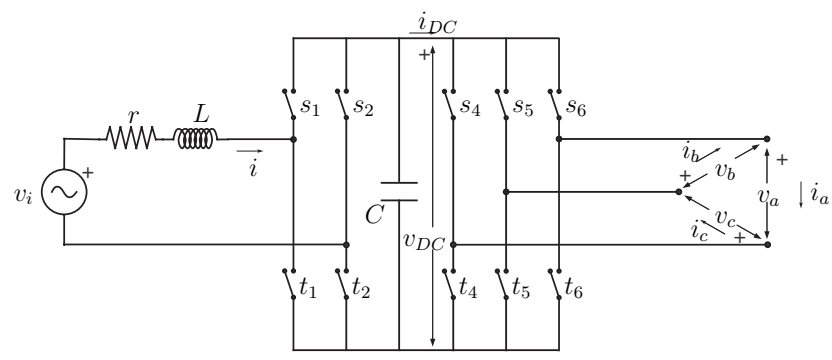

Fig. 3. Back-to-back converter.

$v_{i}(t)=E \sin \left(\omega_{s} t\right)$ is a single-phase AC voltage source, $L$ is the inductance (including the effect of any transformer in the source), $C$ is the capacitor of the DC part, $r$ takes into account all the resistance losses (inductor, source and switches), $s_{k}$ and $t_{k}, k=1,2,4,5,6$. Switch states take values in $\{-1,1\}$ and $t$-switches are complementary to $s$ switches: $t_{k}=\overline{s_{k}}=-s_{k}$. Additionally, $s_{2}=\overline{s_{1}}=-s_{1}$.

The PCHS averaged model of the full-bridge rectifier is as follows. The Hamiltonian variables are (B subindex refers to the B2B subsystem) $x_{B}^{T}=(\lambda, q) \in \mathbb{R}^{2}$, where $\lambda$ is the inductor flux and $q$ is the DC charge in the capacitor. The Hamiltonian function is

$$
H_{B}=\frac{1}{2 L} \lambda^{2}+\frac{1}{2 C} q^{2},
$$

while the structure and damping matrices are

$\mathcal{J}_{B}=\left(\begin{array}{cc}0 & -s_{1} \\ s_{1} & 0\end{array}\right) \in \mathbb{R}^{2 \times 2} \quad \mathcal{R}_{B}=\left(\begin{array}{cc}r & 0 \\ 0 & 0\end{array}\right) \in \mathbb{R}^{2 \times 2}$.

The interconnection matrix is

$g_{B}=\left(\begin{array}{cc}1 & O_{1 \times 3} \\ 0 & f^{T}\end{array}\right) \in \mathbb{R}^{2 \times 4}, \quad f=\frac{1}{2}\left(\begin{array}{c}s_{6}-s_{4} \\ s_{5}-s_{6} \\ s_{4}-s_{5}\end{array}\right) \in \mathbb{R}^{3}$,

with inputs

$$
u=\left(\begin{array}{c}
v_{i} \\
-i_{a b c}
\end{array}\right) \in \mathbb{R}^{4},
$$

where $i_{a b c}^{T}=\left(i_{a}, i_{b}, i_{c}\right) \in \mathbb{R}^{3}$ are the three-phase currents in the inverter part. Notice that the inverter subsystem can be seen as a Dirac structure [3] with

$$
\begin{aligned}
& v_{a b c}=f v_{D C} \\
& i_{D C}=f^{T} i_{a b c}
\end{aligned}
$$

where $v_{a b c}^{T}=\left(i_{a}, i_{b}, i_{c}\right) \in \mathbb{R}^{3}$ are the three-phase voltages and $v_{D C} \in \mathbb{R}$, is the DC voltage, and $i_{D C} \in \mathbb{R}$ is the DC current supplied by the rectifier subsystem.

The bond graph model of the B2B converter is depicted in Fig. 4. The switch model has been taken as a transformer, which has the same behavior than an ideal switch for an averaged model [17].

\section{The dq-transformation}

From an analysis point of view it is convenient to express the three-phase inverter voltages of the DFIM side in $d q$ components [16].

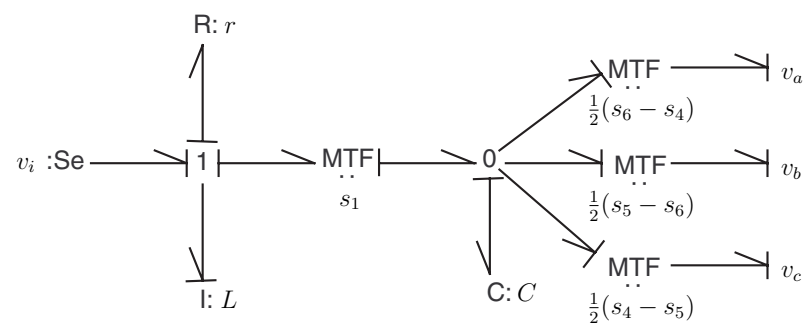

Fig. 4. Bond Graph of the B2B converter.

First, from any set of three phase electrical variables $y_{A B C}$ we compute transformed variables $y_{\alpha \beta \gamma}$ by means of

$$
y_{\alpha \beta \gamma}=T y_{A B C},
$$

with

$$
T=\left(\begin{array}{ccc}
\frac{\sqrt{2}}{\sqrt{3}} & -\frac{1}{\sqrt{6}} & -\frac{1}{\sqrt{6}} \\
0 & \frac{1}{\sqrt{2}} & -\frac{1}{\sqrt{2}} \\
\frac{1}{\sqrt{3}} & \frac{1}{\sqrt{3}} & \frac{1}{\sqrt{3}}
\end{array}\right)
$$

with $T^{T}=T^{-1}$, so that this is a power-preserving transformation $\langle i, v\rangle=\left\langle i_{A B C}, v_{A B C}\right\rangle$. For a three-phase equilibrated system, one has $y_{A}+y_{B}+y_{C}=0$; the $d q$ transformation allows then working with only the two first components (the $\alpha-\beta$ components) and neglect the third one, the $\gamma$, or homopolar, component, which is zero for any balanced set and which, in any case, is dynamically decoupled from the other components.

The second part of the transformation relies on the assumption that the induction machine is symmetric, with a sinusoidal distribution of magnetic fluxes in the air gap. It eliminates the dependence of the equations on $\theta$ (mechanical position of the rotor), and consists in defining new variables $y_{d q}$ via

$$
\begin{gathered}
\left(\begin{array}{c}
y_{\alpha \beta s} \\
y_{\alpha \beta r}
\end{array}\right)=K(\theta, \delta)\left(\begin{array}{l}
y_{d q s} \\
y_{d q r}
\end{array}\right) \\
K(\theta, \delta)=\left(\begin{array}{cc}
e^{J_{2} \delta} & O_{2} \\
O_{2} & e^{J_{2}(\delta-\theta)}
\end{array}\right) \in \mathbb{R}^{4 \times 4}
\end{gathered}
$$

where $\delta$ is an arbitrary function of time, and

$$
e^{J_{2} \phi}=\left(\begin{array}{cc}
\cos (\phi) & -\sin (\phi) \\
\sin (\phi) & \cos (\phi)
\end{array}\right) \in \mathbb{R}^{2 \times 2} .
$$

If $\dot{\delta}$ is the stator frequency $\omega_{s}$, this has the nice additional property of converting the sinusoidal time-dependent stator variables into constant values, which is useful for controlling purposes [12].

The total $A B C \leftrightarrow d q$ transformation can be seen as a Dirac structure defined by

$$
\begin{aligned}
v_{A B C} & =K(\theta, \delta) T v_{d q} \\
i_{d q} & =-(K(\theta, \delta) T)^{T} i_{A B C} .
\end{aligned}
$$

The dq-transformation can be seen, in bond graph terms, as a modulated transformation in two steps, Fig. 5. First the 


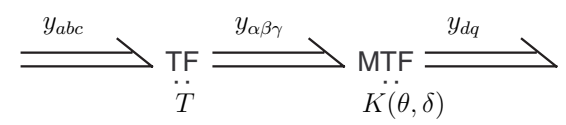

Fig. 5. Bond graph of the $d q$-transformation, using multi-bonds.

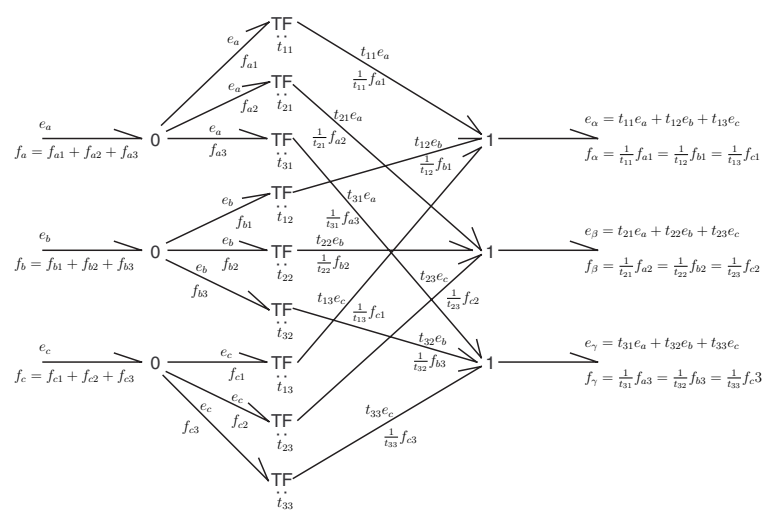

Fig. 6. Bond graph corresponding to the $T$ matrix of the $d q$-transformation.

$T$ transformation reduces, in an equilibrated case, from a 3phase $(a b c)$ to a 2-phase $(\alpha \beta)$. Then $K(\delta, \theta)$ simplifies the dynamical equations of the DFIM.

Fig. 6 shows a completed bond graph of the first step of the $d q$-transformation. Indeed, we write the output equations for efforts

$$
\begin{aligned}
& e_{\alpha}=t_{11} e_{a}+t_{12} e_{b}+t_{13} e_{c} \\
& e_{\beta}=t_{21} e_{a}+t_{22} e_{b}+t_{23} e_{c} \\
& e_{\gamma}=t_{31} e_{a}+t_{32} e_{b}+t_{33} e_{c} .
\end{aligned}
$$

which implement (3). For the flows we have (10)

$$
\begin{gathered}
f_{\alpha}=\frac{1}{t_{11}} f_{a 1}=\frac{1}{t_{12}} f_{b 1}=\frac{1}{t_{13}} f_{c 1} \\
f_{\beta}=\frac{1}{t_{21}} f_{a 2}=\frac{1}{t_{22}} f_{b 2}=\frac{1}{t_{23}} f_{c 2} \\
f_{\gamma}=\frac{1}{t_{31}} f_{a 3}=\frac{1}{t_{32}} f_{b 3}=\frac{1}{t_{33}} f_{c} 3 \\
f_{a}=f_{a 1}+f_{a 2}+f_{a 3} \\
f_{b}=f_{b 1}+f_{b 2}+f_{b 3} \\
f_{c}=f_{c 1}+f_{c 2}+f_{c 3},
\end{gathered}
$$

and finally

$$
f_{a b c}=T^{T} f_{\alpha \beta \gamma},
$$

which, expressed in $\alpha \beta \gamma$-components, is

$$
f_{\alpha \beta \gamma}=\left(T^{T}\right)^{-1} f_{a b c} .
$$

Since $T^{T}=T^{-1}$ we recover expression (3) for flows. Note that for an equilibrated 3-phase system the bond graph takes a form where the $\gamma$-port disappears.

For the second part of the transformation, the bond graph is shown in Fig.7. Following the same steps as for the first part of the transformation, we recover (4), since $e^{-J_{2} \phi}=$ $\left(e^{J_{2} \phi}\right)^{T}$.

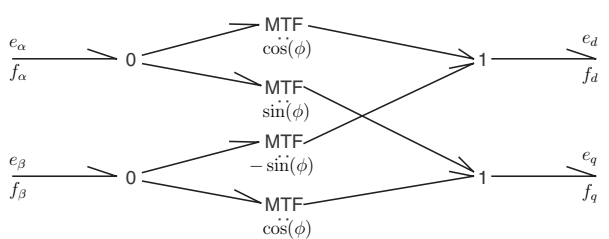

Fig. 7. Bond graph corresponding to the $K$ matrix of the $d q$-transformation
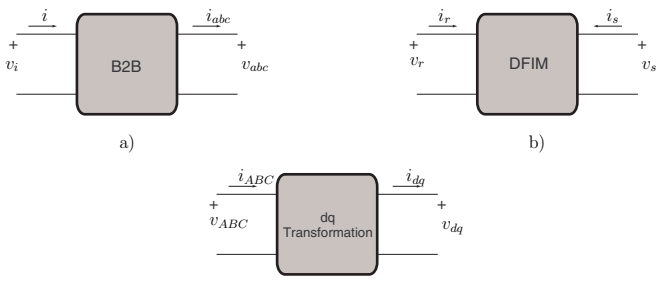

c)

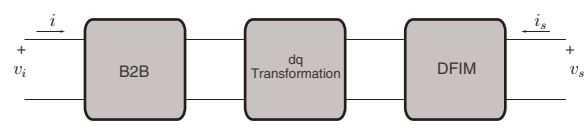

d)

Fig. 8. Interconnection scheme

\section{PCHS model of the whole system}

Fig. 8 shows the interconnection scheme of the whole system (B2B+DFIM). The $d q$-transformation connects the B2B converter with the DFIM as a Dirac structure.

The interconnection relations are

$$
v_{r}=v_{d q}, i_{r}=i_{d q}, v_{A B C}=v_{a b c}, i_{A B C}=i_{a b c} .
$$

We use equations (12) and introduce a new $\mathcal{K}$ matrix

$$
\mathcal{K}=T_{*}^{T} e^{J_{2}(\delta-\theta)} \in \mathbb{R}^{3 \times 2},
$$

with $T_{*}$ defined so as to remove the homopolar component:

$$
T_{*}=\left(\begin{array}{ccc}
\frac{\sqrt{2}}{\sqrt{3}} & -\frac{1}{\sqrt{6}} & -\frac{1}{\sqrt{6}} \\
0 & \frac{1}{\sqrt{2}} & -\frac{1}{\sqrt{2}}
\end{array}\right) \in \mathbb{R}^{2 \times 3} .
$$

The variables of the whole PCHS system are $x^{T}=$ $\left(\Lambda^{T}, J_{m} \omega_{r}, \lambda, q\right) \in \mathbb{R}^{7}$, with energy function

$H=H_{D}+H_{B}=\frac{1}{2} \Lambda^{T} \mathcal{L}^{-1} \Lambda+\frac{1}{2 J_{m}} x_{m}^{2}+\frac{1}{2 L} \lambda^{2}+\frac{1}{2 C} q^{2}$.

The $\mathbb{R}^{7 \times 7}$ structure and dissipation matrices are

$$
\mathcal{J}-\mathcal{R}=\left(\begin{array}{ccccc} 
& & O_{2 \times 1} & O_{2 \times 1} \\
\mathcal{J}_{D}-\mathcal{R}_{D} & & O_{2 \times 1} & \mathcal{K}^{T} f \\
& & 0 & 0 \\
O_{1 \times 2} & O_{1 \times 2} & 0 & \mathcal{J}_{B}-\mathcal{R}_{B} \\
O_{1 \times 2} & -f^{T} \mathcal{K} & 0 &
\end{array}\right),
$$

and the interconnection matrix and port variables are

$$
g=\left(\begin{array}{cc}
I_{2} & O_{2 \times 1} \\
O_{2} & O_{2 \times 1} \\
O_{1 \times 2} & 0 \\
O_{1 \times 2} & 1 \\
O_{1 \times 2} & 0
\end{array}\right) \in \mathbb{R}^{7 \times 3} \quad u^{T}=\left(v_{s}^{T}, v_{i}\right) \in \mathbb{R}^{3} .
$$

The bond graph of the whole system is shown in Fig. 9. 


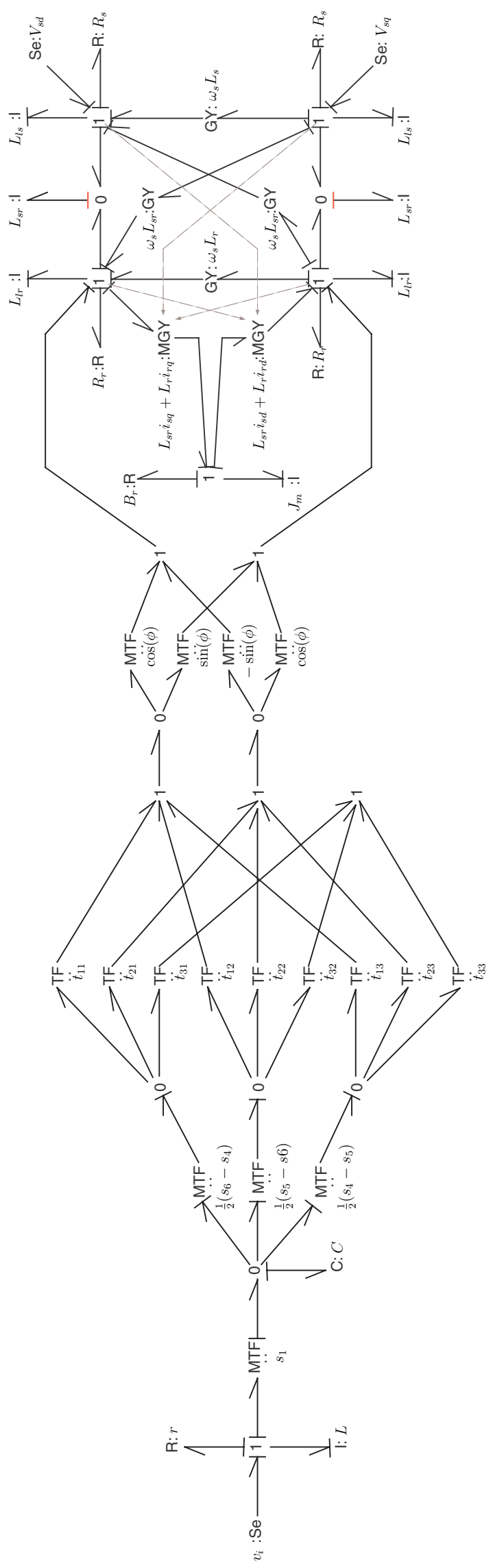

Fig. 9. Bond graph representation of the whole system.

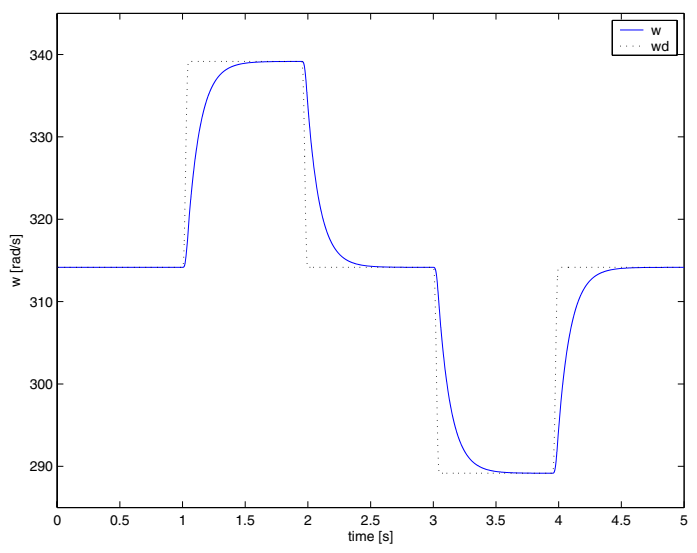

Fig. 10. Mechanical speed $\omega_{r}$.

\section{SIMULATIONS}

In this Section we implement a numerical simulation of the whole system controlled via the IDA-PBC controllers [10], using two controllers designed for the DFIM and the B2B in [12] and [18], respectively. The simulation has been performed using the 20 -sim ${ }^{1}$ modeling and simulation software. The parameters used in the simulations are given in Table I. For the purposes of testing the model, the desired

\begin{tabular}{|c|c|c|c|c|c|c|c|}
\hline DFIM & $L_{s r}$ & $L_{r}, L_{s}$ & $J_{m}$ & $B_{r}$ & $R_{s}$ & $R_{r}$ & $v_{g}$ \\
\hline Value & 0.041 & 0.042 & 0.0005 & 0.005 & 0.087 & 0.0228 & $(380,0)$ \\
\hline & B2B & $r$ & $\bar{L}$ & & & $E$ & \\
\hline & Value & 0.08 & $1 \cdot 10^{-3}$ & 4.5 . & $0^{-3}$ & 68.16 & \\
\hline
\end{tabular}

SIMULATION PARAMETER VALUES (IN SI UNITS) FOR THE DFIM AND THE B2B. AdDitionally, $\omega_{s}=\omega_{o}=2 \pi 50$.

mechanical speed changes around $\omega=2 \cdot 50 \pi$ (dotted line in Fig. 10) and a desired bus voltage $v_{D C}^{*}=150$ has been selected.

Fig. 10 shows the desired and simulated mechanical speed. Fig. 11 shows the reactive power compensation of the stator side of the DFIM. Fig. 12 shows $v_{D C}$, which remains close to the desired value even in the transient of the machine. The small oscillations of the DC-link voltage are intrinsic to the system, due its 0-dynamics [19]. Finally, voltage $v_{i}$ and current $i$ at the single phase source feeding the B2B are depicted in Fig. 13, showing that they are nearly in phase. The small phase shift is due to the fact that the controller designed in [18] disregards higher harmonics (for example the second harmonic component of the DC-link voltage).

\section{CONCLUSIONS}

We have modeled and simulated a complex system, with several subsystems from the electric, power electronics and mechanical domains, using both the PCHS and the Bond Graph paradigms. The simulations have been run in closed

\footnotetext{
${ }^{1}$ See www. 20 sim.com
} 


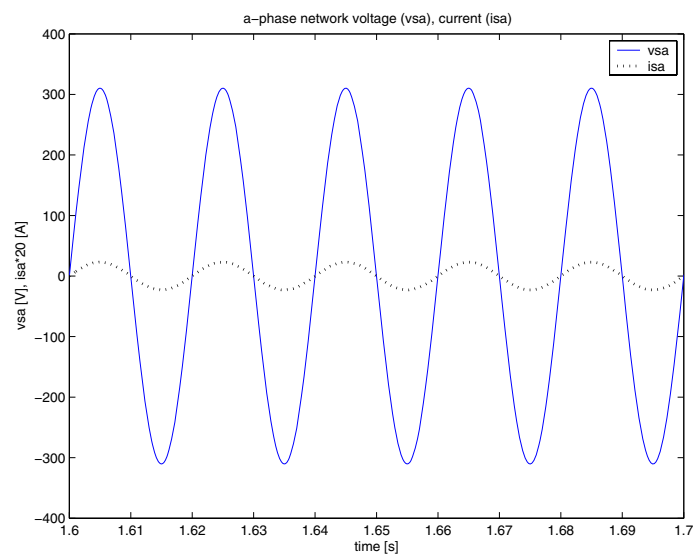

Fig. 11. Detail of the grid $a$-phase voltage $v_{s a}$ and current $i_{s a}$.

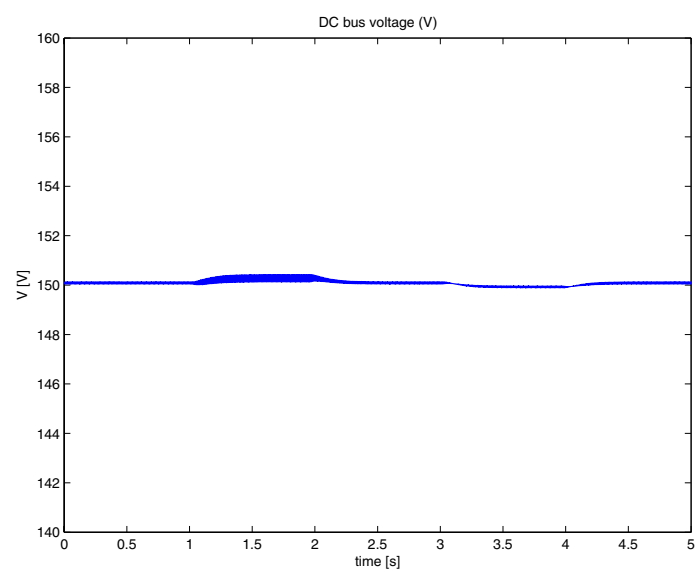

Fig. 12. DC-bus voltage $v_{D C}$ of the B2B.

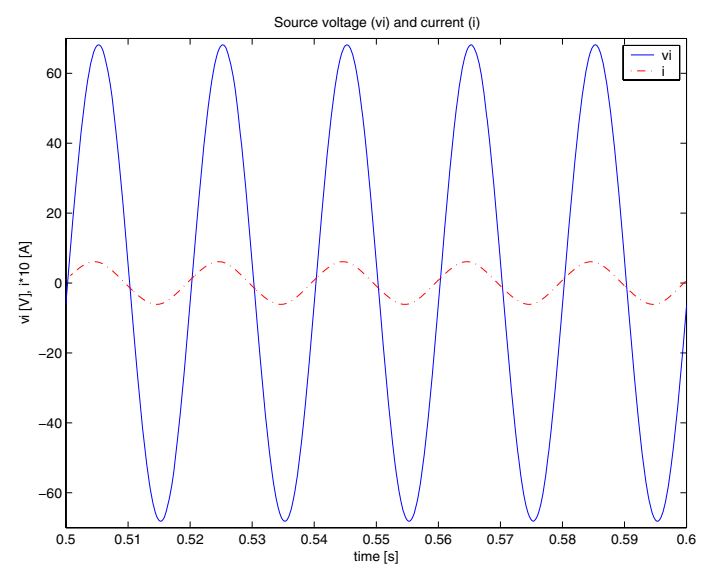

Fig. 13. Detail of the AC single-phase voltage and current for the B2B. loop with controllers designed with the IDA-PBC technique developed for port Hamiltonian systems. The description of the whole system as a network of interconnected subsystems which exchange power in a preserving way has proved itself useful both from the modelling and the control specification and design points of view.

\section{REFERENCES}

[1] B. Maschke and A. van der Schaft, "Port-controlled Hamiltonian Systems: Modelling origins and system-theoretic properties," in 2nd. IFAC NOLCOS, 1992, pp. 282-288.

[2] _ Systems and networks: Mathematical theory and applications. Academic-Verlag, 1994, vol. II, pp. 349-352.

[3] M. Dalsmo and A. van der Schaft, "On representations and integrability of mathematical structures in energy-conserving physical systems," SIAM J. Control Optim., vol. 37, pp. 54-91, 1998.

[4] B. Maschke, A. van der Schaft, and P. C. Breedveld, "An intrinsic hamiltonian formulation of the dynmics of lc-circuits," IEEE Trans. Circuits and Syst., vol. 42, pp. 73-82, 1995.

[5] P. Breedveld, "MultiBond-graph elements in physical systems theory," Journal of the Franklin Institute, vol. 319, pp. 1-36, 1985.

[6] G. Golo, A. van der Schaft, P. Breedveld, and B. Maschke, "Hamiltonian formulation of Bond Graphs," in Workshop NACO II, 2001, pp. 2642-2647.

[7] A. van der Schaft and B. Maschke, "Hamiltonian formulation of distributed-parameter systems with boundary energy flow," Journal of Geometry and Physics, vol. 42, pp. 166-194, 2002.

[8] A. Kugi, Non-linear control based on physical models. Springer, 2001.

[9] A. van der Schaft, $L_{2}$ gain and passivity techniques in nonlinear control. Springer, 2000.

[10] R. Ortega, A. van der Schaft, B. Maschke, and G. Escobar, "Interconnection and damping assignment passivity-based control of portcontrolled Hamiltonian systems," Automatica, vol. 38, no. 4, pp. 585 596, 2002.

[11] D. Karnopp, D. Margolis, and R. Rosenberg, System dynamics modeling and simulation of mechatronic systems, 3rd ed. J. Wiley, New York, 2000

[12] C. Batlle, A. Dòria-Cerezo, and R. Ortega, "Power Flow Control of a Doubly-Fed Induction Machine Coupled to a Flywheel," European Journal of Control, vol. 11, no. 3, pp. 209-221, 2005.

[13] R. Peña, J. C. Clare, and G. M. Asher, "Doubly fed induction generator using back-to-back pwm converters and its application to variable speed wind-energy generation," in IEEE Proc. Electric Power Applications, vol. 143-5, 1996, pp. 231-241.

[14] P. Caratozzolo, E. Fossas, J. Pedra, and J. Riera, "Dynamic modeling of an isolated motion system with DFIG," in Proc. CIEP, 2000, pp. 287-292.

[15] S. Peresada, A. Tilli, and A. Tonelli, "Power control of a doubly fed induction machine via output feedback," Control Engineering Practice, vol. 12, pp. 41-57, 2004

[16] P. C. Krause, Analysis of electric machinery. McGraw-Hill, 1986.

[17] M. Delgado and H. Sira-Ramirez, "Modeling and simulation of a switch regulated DC-to-DC power converters of the boost type," in IEEE Proc. Conf. on Devices, Circuits and Systems, 1995, pp. 84-88.

[18] C. Batlle, A. Dòria-Cerezo, and E. Fossas, "IDA-PBC controller for a bidirectional power flow full-bridge rectifier," in IEEE Proc. Conference on Decision and Control, 2005.

[19] R. Griñó, E. Fossas, and D. Biel, "Sliding mode control of a full-bridge unity power factor rectifier,' Lecture Notes in Control and Information Sciences, vol. 281, pp. 139-148, 2002. 